\title{
Cromosoma 13 en anillo
}

\author{
Francisco Cammarata-Scalisi ${ }^{1 *}$, Yajaira Briceño ${ }^{2}$, Edymar Cegarra ${ }^{3}$ y Daniela Montilla ${ }^{4}$ \\ ${ }^{1}$ Unidad de Genética Médica, Departamento de Puericultura y Pediatría, Facultad de Medicina, Universidad de Los Andes; ${ }^{2}$ Unidad de \\ Endocrinología, Instituto Autónomo Hospital Universitario de Los Andes; ${ }^{3}$ Posgrado de Puericultura y Pediatría, Universidad de Los Andes; ${ }^{4}$ Departamento \\ de Microbiología y Parasitología, Cátedra de Inmunología, Facultad de Farmacia y Bioanálisis, Universidad de Los Andes. Mérida, Venezuela
}

\section{Resumen}

Introducción: El cromosoma 13 en anillo es una alteración citogenética infrecuente, clínicamente caracterizada por presentar retraso del crecimiento, del desarrollo psicomotor y déficit cognitivo, además de microcefalia, dismorfia facial, alteraciones genitales e hipoplasia del pulgar. Caso clínico: Paciente de 8 meses de edad evaluado por presentar talla baja, retraso del desarrollo psicomotor, microcefalia, dismorfia facial, hipospadias peneoescrotales e hipoplasia de pulgar. Se evidenció lisencefalia, hipoacusia neuroconductiva del lado derecho y comunicación interauricular tipo ostium secundum pequeña. El estudio citogenético del paciente mostró 46, XY, r (13) en 30 células analizadas. Conclusiones: Se resaltan los hallazgos clínicos que pueden orientar el diagnóstico de esta alteración cromosómica estructural infrecuente, destacando además, la evaluación médica interdisciplinaria requerida y el adecuado asesoramiento genético familiar.

Palabras clave: Cromosoma 13 en anillo. 46, XY, r (13). Clínica. Asesoramiento genético.

\section{Ring chromosome 13}

\section{Abstract}

Background: Ring chromosome 13 is an infrequent cytogenetic disorder clinically characterized by growth and psychomotor development retardation, cognitive deficit, microcephaly, facial dysmorphism, genital alterations and thumb hypoplasia. Case report: A 8-month-old patient was evaluated for presenting short stature, psychomotor development delay, microcephaly, facial dysmorphism, penoscrotal hypospadias and thumb hypoplasia. Lissencephaly, neuroconductive hearing loss on the right side and small ostium secundum interatrial communication were evident. The cytogenetic study of the patient showed 46, XY, r (13) in 30 cells analyzed. Conclusions: Clinical findings that can guide the diagnosis of this infrequent structural chromosomal alteration are highlighted, as well as the interdisciplinary medical evaluation required and adequate family genetic counseling.

Key words: Ring chromosome 13. 46, XY, r (13). Clinic. Genetic counseling.

Disponible en internet: 18-03-2019 Bol Med Hosp Infant Mex. 2019;76:100-103 www.bmhim.com 


\section{Introducción}

Un cromosoma en anillo ocurre después de la rotura en ambos brazos de un cromosoma, seguida de la fusión de los extremos rotos para completar el anillo $0^{1-3}$. Aunque infrecuente, este tipo de alteración citogenética se ha descrito en cada cromosoma humano ${ }^{4}$. Las características clínicas observadas en esta alteración varían dependiendo del número y función de los genes que presentan deleción, duplicación y la estabilidad del anillo'.

El cromosoma 13 en anillo es una alteración citogenética infrecuente descrita por primera vez en 1968 por Lejeune, et al. ${ }^{2,5}$ Presenta una incidencia estimada de 1 en 58,000 nacidos vivos ${ }^{1,5,6}$ y es más común $(60 \%)$ en el sexo femenino ${ }^{6}$. La mayoría de los casos informados en la literatura son de novo ${ }^{1,2}$; sin embargo, se han notificado casos hereditarios ${ }^{1,4}$. Las características clínicas incluyen retraso del crecimiento intrauterino, defecto de cierre del tubo neural, alteraciones en el sistema nervioso central ${ }^{5}$, convulsiones ${ }^{3}$, retraso del desarrollo psicomotor ${ }^{2}$, déficit cognitivo de moderado a grave, deficiencia del crecimiento, microcefalia ${ }^{1-3,5,6}$, alteraciones oculares, hipoacusia ${ }^{3}$, dismorfia facial caracterizada por cabello de implantación anterior alta, hipertelorismo, fisuras palpebrales hacia abajo y afuera, puente nasal ancho, labios delgados y microstomía, además de cardiopatía congénita ${ }^{3,6}$, alteraciones genitales en el sexo masculino que incluyen criptorquidia e hipospadias $^{1,2,5}$, atresia anal ${ }^{3}$ e hipoplasia de pulgar $^{1}$ (Tabla 1). Estas características pueden ser similares 0 más graves ${ }^{2,5,7}$ que las observadas en los portadores de la deleción del brazo largo del cromosoma 13.

La gravedad clínica encontrada en los casos de cromosoma 13 en anillo es amplia ${ }^{3,6}$. La pérdida de genes a partir de $13 q 34$ puede contribuir al fenotipo ${ }^{5}$. No obstante, la correlación genotipo-fenotipo no se ha identificado claramente ${ }^{2}$.

El objetivo del presente trabajo es informar un caso de cromosoma 13 en anillo y los diferentes hallazgos clínicos que pueden orientar su diagnóstico, así como resaltar la importancia de brindar una evaluación médica interdisciplinaria y un adecuado asesoramiento genético.

\section{Caso clínico}

Paciente de sexo masculino de 8 meses de edad evaluado por presentar retraso del desarrollo psicomotor, talla baja, microcefalia, dismorfia facial e hipospadias peneoescrotales.
Tabla 1. Hallazgos clínicos del síndrome del cromosoma 13 en anillo

\begin{tabular}{l|c}
$\begin{array}{l}\text { Hallazgos reportados en } \\
\text { literatura }{ }^{-3,5,6}\end{array}$ & $\begin{array}{c}\text { Hallazgos en el } \\
\text { paciente } \\
\text { (12 meses de edad) }\end{array}$
\end{tabular}

Defecto de cierre de tubo neural

Retraso del crecimiento intrauterino

Talla baja

Alteraciones en el sistema nervioso

Microcefalia

(12 meses de edad)

Cabello de implantación anterior alta

Prominencia frontal

Alteraciones oculares

Hipertelorismo

Fisura palpebral hacia arriba y afuera

Puente nasal ancho y deprimido

Labios delgados

Microstomía

Incisivos prominentes

Hipoacusia

Pabellones auriculares prominentes

Hipotiroidismo

Cardiopatía congénita

Alteraciones renales

Genitales ambiguos

Hipospadias

Criptorquidia

Atresia anal

Hipoplasia de pulgares

Déficit cognitivo

NP

Retraso en el lenguaje

$+$

Autismo

NP

Convulsiones

Retinoblastoma

Disminución en la sobrevida

NP

+: presente; -: ausente; NP: no precisado (hallazgo limitado por la edad).

En el momento de la concepción, el padre tenía 23 años de edad y la madre, 19, ambos sanos y no consanguíneos. Producto de primera gestación. Embarazo 
con infección de las vías urinarias al sexto mes, que fue tratada. Diagnóstico prenatal de retraso de crecimiento intrauterino. El paciente nació por cesárea a las 35 semanas de gestación, sin complicaciones, con un peso al nacer de 2,200 g (desviación estándar [DE]: $-2.7)$ y una talla de $45 \mathrm{~cm}$ (DE: -4.1$)$.

A los 12 meses, talla de $67 \mathrm{~cm}$ (DE: -3.6), peso de $6.9 \mathrm{~kg}(\mathrm{DE}-3.6)$ y perímetro cefálico de $39.5 \mathrm{~cm}$ (percentil <3). Presentaba microcefalia, cabello de implantación anterior alta, región frontal amplia y prominente, puente nasal ancho y deprimido, punta nasal bulbosa, columela corta, labio superior en forma de arco de cupido e inferior grueso, microstomía, micrognatia y cuello corto (Fig. 1A). Se detectó tórax normoexpansible con soplo sistólico, abdomen depresible y no doloroso. A nivel genital presentó hipospadias peneoescrotales. En las extremidades se evidenció hipoplasia bilateral de pulgares. El paciente se encontraba neurológicamente activo con adecuado tono muscular.

La tomografía axial computarizada evidenció lisencefalia. La evaluación por oftalmología no encontró errores de refracción ni alteraciones en el segmento anterior, cristalino y en el fondo de ojo. El estudio de potenciales evocados auditivos en el tallo cerebral fue anormal del lado derecho, lo que sugiere hipoacusia neuroconductiva; el estudio fue normal del lado izquierdo. El ecocardiograma transtorácico evidenció comunicación interauricular tipo ostium secundum pequeña sin repercusión hemodinámica. La ecografía renal mostró riñón ectópico izquierdo, pielectasia renal izquierda y microlitiasis renal bilateral. La radiografía de mano del paciente evidenció hipoplasia de pulgar (Fig. 1B). El estudio citogenético del paciente mostró 46, XY, r (13) en 30 células analizadas (Fig. 1C). Los padres no presentaron alteraciones cromosómicas.

La evolución clínica del paciente fue satisfactoria, a pesar de haber cursado con anemia leve e infecciones respiratorias recurrentes que ameritaron hospitalizaciones a los 14 y 17 meses de edad. Los controles de perfil tiroideo, así como otros parámetros bioquímicos, se encontraron dentro de los valores normales.

\section{Discusión}

Se han descrito tres diferentes tipos del síndrome del cromosoma 13 en anillo, según la ubicación de los puntos de corte en el brazo largo del cromosoma 13 durante la formación del cromosoma en anillo ${ }^{2,8}$. El primer grupo es el más frecuente ${ }^{8}$ e involucra la pérdida de los segmentos $13 q 34$ y 13q33 2,8 . Se caracteriza por déficit cognitivo, microcefalia, prominencia frontal, hipertelorismo,

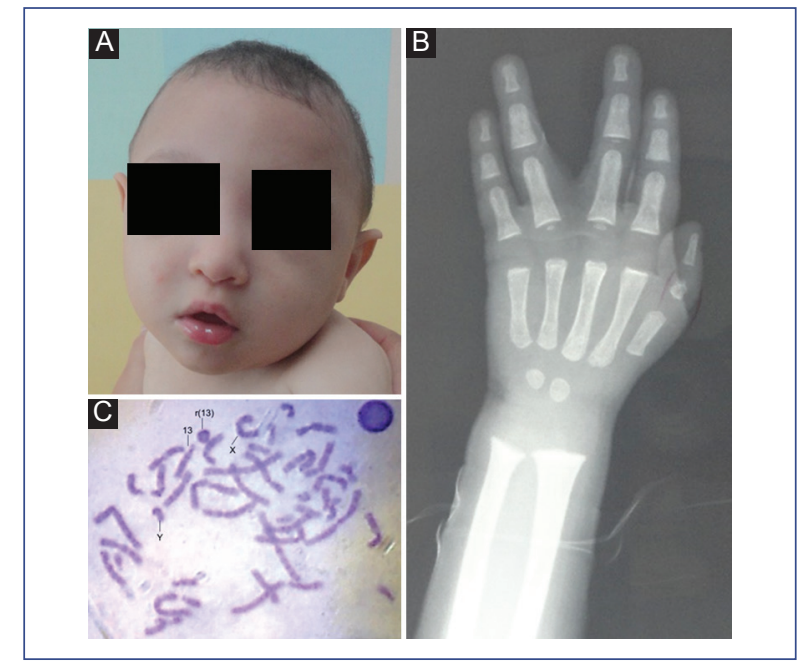

Figura 1. A: se observa microcefalia, cabello escaso con implantación anterior alta, región frontal amplia, punta nasal bulbosa y microstomía en el paciente. B: en la radiografía de mano se observa hipoplasia de pulgar. C: el estudio citogenético señala los cromosomas sexuales (X y Y), además de los dos cromosomas 13 , uno de ellos en anillo ( $r 13)$.

puente nasal deprimido, dientes incisivos sobresalientes, pabellones auriculares grandes ${ }^{2}$ y retraso en el crecimiento $^{6}$. El segundo grupo resulta de la pérdida de los segmentos $13 q 32$ y $13 q 31^{2,8}$, y se caracteriza por los hallazgos del primer grupo, además de aplasia de los pulgares y pies, anomalías genitales, atresia anal y malformaciones oculares. Finalmente, el tercer grupo resulta de la pérdida del segmento 13q21, y presenta retinoblastoma como hallazgo característico ${ }^{2}$.

Los estudios de microarreglos han sido útiles para definir los puntos de corte y tamaño de la deleción ${ }^{3}$. Aunque el paciente estudiado no cuenta con este tipo de análisis, los hallazgos clínicos sugieren que se encontraría en el segundo grupo, ya que presenta hipospadias e hipoplasia de pulgares.

Se ha presentado hipotiroidismo en pacientes con cromosoma 13 en anillo². En este caso, se descartó dicha alteración con la pesquisa neonatal y dos controles posteriores que se encontraron dentro de la normalidad. La hipoacusia y el retardo en el lenguaje son hallazgos comunes $^{5}$ y se encuentran presentes en el paciente estudiado. Por otra parte, el autismo se ha descrito en algunos casos y se encuentra asociado con otras alteraciones neurológicas, como déficit cognitivo y convulsiones ${ }^{1}$. Por la corta edad del paciente, algunos de estos hallazgos no se pueden determinar. Ante la variada forma de presentación clínica de esta entidad, el 
seguimiento médico es necesario para descartar y tratar, en lo posible, los hallazgos que pueden presentarse.

Los pacientes que muestran deleciones en el brazo largo del cromosoma 13 pueden cursar con genitales ambiguos o hipospadias, lo que sugiere que en esta región se encuentran genes importantes para el desarrollo genital ${ }^{2}$. Además, en $13 q 34$ se encuentran los genes $F 7$ y $F 10$, que codifican los factores de coagulación VII (FVII) y X (FX), respectivamente. Por lo tanto, los niveles de FVII y FX en los pacientes con cromosoma 13 en anillo se pueden encontrar disminuidos hasta en el $50 \%$ en comparación con un individuo sin la alteración, por lo que estos pacientes pueden cursar con efectos menores en los tiempos de coagulación ${ }^{6}$.

El diagnóstico prenatal es infrecuente. Los casos han sido informados a través de estudios de amniocentesis en pacientes que han cursado con defecto de cierre de tubo neural ${ }^{9}$. Aunque en el caso que se reporta no se realizó el diagnóstico prenatal, el retraso del crecimiento intrauterino se puede asociar con la presencia de cromosomopatía.

Las características clínicas de este tipo de anomalía son muy variadas y, ante ello, se sugiere la necesidad de realizar una evaluación médica interdisciplinaria. EI caso clínico informado corresponde al cromosoma 13 en anillo en una presentación de novo, ya que los estudios citogenéticos realizados a los padres fueron normales. No obstante, se debe considerar el riesgo de mosaicismo germinal en ambos padres y, en este caso, la recurrencia es de hasta el $1 \%{ }^{10}$. Con esto, se permite realizar un adecuado y oportuno asesoramiento genético familiar. Finalmente, se recomienda una evaluación exhaustiva prenatal para descartar alteraciones en futuras gestaciones de los progenitores.

\section{Responsabilidades éticas}

Protección de personas y animales. Los autores declaran que para esta investigación no se han realizado experimentos en seres humanos, ni en animales.

Confidencialidad de los datos. Los autores declaran que han seguido los protocolos de su centro de trabajo sobre la publicación de datos de pacientes.
Derecho a la privacidad y consentimiento informado. Los autores han obtenido el consentimiento informado de los pacientes o sujetos referidos en el artículo. Este documento obra en poder del autor de correspondencia.

\section{Conflicto de intereses}

Los autores declaran no tener ningún conflicto de intereses.

\section{Financiamiento}

Ninguno.

\section{Agradecimientos}

A la licenciada Rosalia Gumina, Directora de la Biblioteca del Instituto Autónomo Hospital Universitario de Los Andes, Universidad de Los Andes.

\section{Bibliografía}

1. Charalsawadi C, Maisrikhaw W, Praphanphoj V, Wirojanan J, Hansakunachai $\mathrm{T}$, Roongpraiwan $\mathrm{R}$, et al. A case with a ring chromosome 13 in a cohort of 203 children with non-syndromic autism and review of the cytogenetic literature. Cytogenet Genome Res. 2014;144:1-8.

2. Ozsu E, Yeşiltepe Mutlu G, Ipekçi B. Ring chromosome 13 and ambiguous genitalia. J Clin Res Pediatr Endocrinol. 2014;6:122-4.

3. Kaylor J, Alfaro M, Ishwar A, Sailey C, Sawyer J, Zarate YA. Molecular and cytogenetic evaluation of a patient with ring chromosome 13 and discordant results. Cytogenet Genome Res. 2014;144:104-8.

4. Bedoyan JK, Flore LA, Alkatib A, Ebrahim SA, Bawle EV. Transmission of ring chromosome 13 from a mother to daughter with both having a 46,XX, r(13)(p13q34) karyotype. Am J Med Genet A. 2004;129A:316-20.

5. Abdallah-Bouhjar IB, Mougou-Zerelli S, Hannachi H, Gmidène A, Labalme A, Soyah N, et al. Molecular cytogenetic and phenotypic characterization of ring chromosome 13 in three unrelated patients. J Pediatr Genet. 2013;2:147-55.

6. Su PH, Chen CP, Su YN, Chen SJ, Lin LL, Chen JY. Smallest critical region for microcephaly in a patient with mosaic ring chromosome 13. Genet Mol Res. 2013;12:1311-7.

7. Sankar VH, Phadke SR. Ring chromosome 13 in an infant with ambiguous genitalia. Indian Pediatr. 2006;43:258-60.

8. Brandt CA, Hertz JM, Petersen MB, Vogel F, Noer H, Mikkelsen M. Ring chromosome 13: lack of distinct syndromes based on different breakpoints on 13q. J Med Genet. 1992;29:704-8.

9. Chen CP, Tsai CH, Chern SR, Wu PS, Su JW, Lee CC, et al. Prenatal diagnosis and molecular cytogenetic characterization of mosaic ring chromosome 13. Gene. 2013;529:163-8.

10. Firth HV, Hurst JA. Desk reference: clinical genetics. Oxford: Oxford University Press; 2005. 